\title{
О РАСЧЕТЕ ПОЛОГИХ ОБОЛОЧЕК ОТРИЦАТЕЛЬНОИ КРИВИЗНЫ, СОПРОТИВЛЯЮЩИХСЯ ТОЛЬКО РАСТЯЖЕНИЮ
}

В последнее время безмоментные оболочки отрицательной кривизны, в частности висячие покрытия, находят все более широкое распространение. Благодаря этому значительно возрос интерес к вопросам статического расчета таких оболочек. В разработке инженерных методов расчета висячих покрытий достигнуты определенные успехи. Появился целый ряд трудов, посвященных различным аспектам работы указанных систем (см. $[2-6])$. Все же обычно не удается учесть весь комплекс факторов, оказывающих влияние на их работу, хотя вследствие слабой связности системы такой учет представляется весьма важным.

В настоящей работе выведены основные уравнения для пологих оболочек отрицательной кривизны, сопротивляющихся только растяжению. Рассматривается упругая стадия работы оболочки и принимаются обычные допущения, вытекающие из условия пологости. Учитывается геометрическая нелинейность задачи, а также нелинейность, обусловленная предварительным напряжением системы. Дается возможный путь решения полученных уравнений и приводятся некоторые соображения о влиянии ряда факторов на результаты расчета.

Уравнения равновесия в исходном состоянии. Из поверхности пологой оболочки выделяем элемент, грани которого расположены в плоскостях, параллельных координатным поверхностям $x z$ и $y z$ (рис. 1).
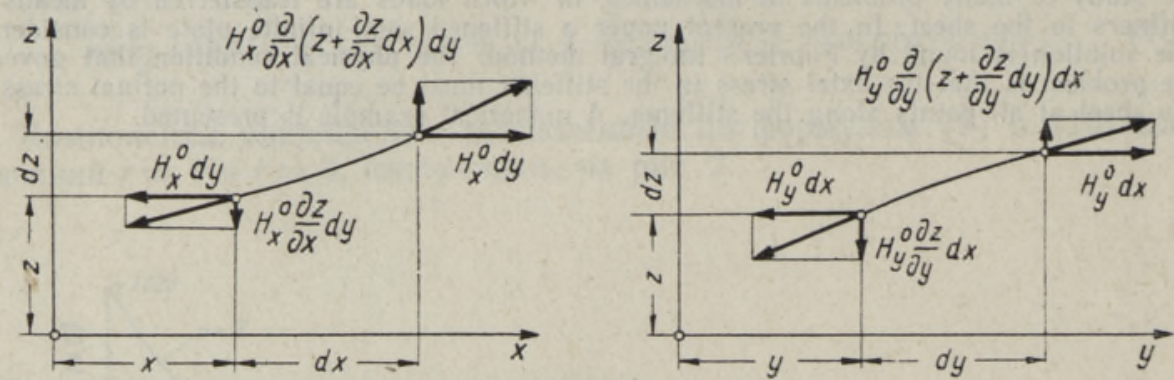

Рис. 1.

Поскольку рассматриваемая оболочка сопротивляется только растяжению, усилия, действующие на грани выделяемого элемента, должны быть перпендикулярны грани элемента и направлены по касательной к поверхности оболочки. Вследствие пологости оболочки можно считать, что указанное усилие не имеет составляющей в направлении касательной к грани элемента. Разлагаем усилия предварительного напряжения на вертикальные и горизонтальные составляющие, обозначая их соответственно $H_{x}^{0}$ и $H_{y}^{0}$. Тогда соответствующие вертикальные составляющие будут равны

$$
H_{x}^{0} \frac{\partial z}{\partial x} \quad \text { и } \quad H_{y}^{0} \frac{\partial z}{\partial y} .
$$

Уравнения равновесия исходного состояния можем представить следующим образом: 


$$
\begin{aligned}
& \Sigma x=0: \frac{\partial H_{x}^{0}}{\partial x}=0 \\
& \Sigma y=0: \frac{\partial H_{y}^{0}}{\partial y}=0 \\
& \Sigma z=0: H_{x}^{0} \frac{\partial^{2} z}{\partial x^{2}}+H_{y}^{0} \frac{\partial^{2} z}{\partial y^{2}}=0 .
\end{aligned}
$$

Распределение усилий предварительного напряжения одного направления, например $H_{x}^{0}=Y_{0}(y)$, должно быть задано по соображениям обеспечения геометрической неизменяемости системы. Тогда начальные усилия другого направления $H_{y}^{0}=X_{0}(x)$ определяются уравнением равновесия (3) в зависимости от формы оболочки. Интересно отметить, что в случае гиперболического параболоида для любой точки оболочки в исходном состоянии

$$
H_{y}^{0}=k H_{x}^{0}=\text { const. }
$$

Уравнения равновесия при действии внешней нагрузки. Внешние нагрузки вызывают определенные смещения поверхности оболочки. В общем случае в соответствующие уравнения равновесия должны войти геометрические показатели деформированной поверхности оболочки. Усилия, действующие на элемент оболочки изображены на рис. 2 .
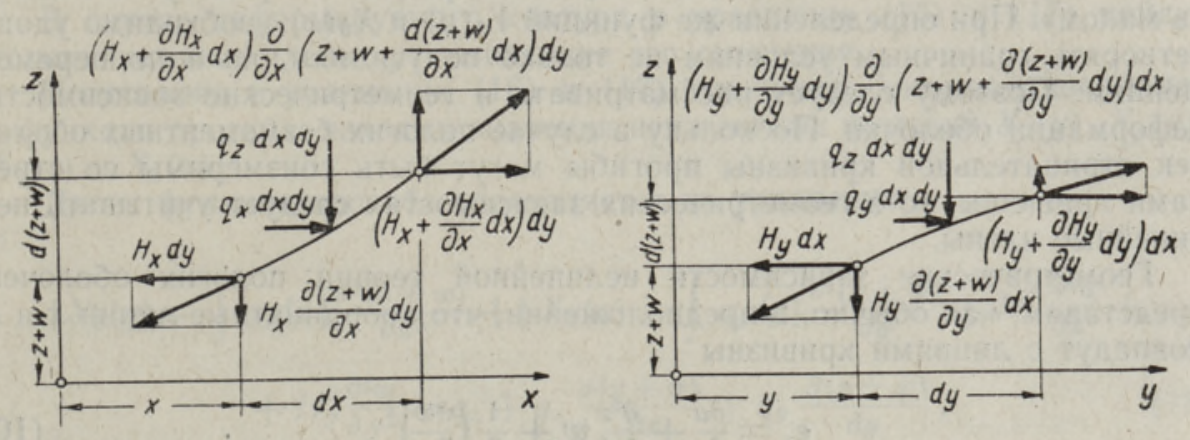

Рис. 2.

Уравнения равновесия можем представить аналогично предыдущему:

$$
\begin{gathered}
\Sigma x=0: \frac{\partial H_{x}}{\partial x}+q_{x}=0 \\
\Sigma y=0: \frac{\partial H_{y}}{\partial y}+q_{y}=0 \\
\Sigma z=0: H_{x} \frac{\partial^{2}(z+w)}{\partial x^{2}}+\frac{\partial H_{x}}{\partial x} \frac{\partial(z+w)}{\partial x}+\frac{\partial H_{x}}{\partial x} d x \frac{\partial^{2}(z+w)}{\partial x^{2}}+ \\
+H_{y} \frac{\partial^{2}(z+w)}{\partial y^{2}}+\frac{\partial H_{u}}{\partial y} \frac{\partial(z+w)}{\partial y}+\frac{\partial H_{u}}{\partial y} d y \frac{\partial^{2}(z+w)}{\partial y^{2}}- \\
-q_{z}-q_{x} \frac{\partial(z+w)}{\partial x}-q_{y} \frac{\partial(z+w)}{\partial y}=0
\end{gathered}
$$

где $w-$ функция прогиба. 
Далее введем обозначения

$$
\Delta H_{x}=H_{x}-H_{x}{ }^{0} \text { и } \Delta H_{y}=H_{y}-H_{y}{ }^{0} .
$$

Тогда уравнения равновесия (4) и (5) с учетом уравнений (1) и (2) после интегрирования принимают вид

$$
\begin{aligned}
\Delta H_{x} & =Y_{0}(y)-\int q_{x} d x \\
\Delta H_{y} & =X_{0}(x)-\int q_{u} d y,
\end{aligned}
$$

где функции $Y_{0}(y)$ и $X_{0}(x)$ определяются из граничных условий.

У равнение (6) после исключения малых высшего порядка с учетом зависимости (3) может быть представлено в виде

$$
\begin{gathered}
\Delta H_{x} \frac{\partial^{2}(z+w)}{\partial x^{2}}+\Delta H_{y} \frac{\partial^{2}(z+w)}{\partial y^{2}}+H_{x}^{0} \frac{\partial 2 w}{\partial x^{2}}+H_{y}^{0} \frac{\partial^{2} w}{\partial y^{2}}- \\
-q_{z}-q_{x} \frac{\partial(z+w)}{\partial x}-q_{y} \frac{\partial(z+w)}{\partial y}=0 .
\end{gathered}
$$

Геометрические зависимости. Уравнения (7), (8) и (9) с учетом уравнений начального состояния полностью определяют работу оболочки «в малом». При определении же функций $Y_{0}(y)$. и $X_{0}(x)$ необходимо удовлетворять граничным условиям не только по усилиям, но и по перемещениям. Поэтому следует рассматривать и геометрические зависимости деформации оболочки. Поскольку в случае пологих безмоментных оболочек отрицательной кривизны прогибы могут быть соизмеримы со стрелами кривизны, то в геометрических зависимостях следует учитывать нелинейные члены.

Геометрические зависймости нелинейной теории пологих оболочек представим, как обычно, в предположении, что координатные линии $x$ и $u$ совпадут с линиями кривизны

$$
\begin{aligned}
& \varepsilon_{x}=\frac{\partial u}{\partial x}+\frac{\partial^{2} z}{\partial x^{2}} w+\frac{1}{2}\left(\frac{\partial w}{\partial x}\right)^{2} \\
& \varepsilon_{y}=\frac{\partial v}{\partial y}+\frac{\partial^{2} z}{\partial y^{2}} w+\frac{1}{2}\left(\frac{\partial w}{\partial y}\right)^{2},
\end{aligned}
$$

где $u$ и $v$ - тангенциальные смещения точек оболочки; $\varepsilon_{x}$ и $\varepsilon_{y}-$ соответствующие относительные удлинения.

Схема решения задачи. Как было указано выше, деформированное состояние оболочки определяется уравнениями (7), (8) и (9). Для исключения из этих уравнений составляющих усилий $\Delta H_{x}$ и $\Delta H_{y}$ используем геометрические зависимости (10) и (11) и физические уравнения. Поскольку в оболочках типа тросовых висячих покрытий коэффициент Пуасона равен нулю, зависимости между усилиями и соответствующими удлинениями выражаются простыми законами Гука

$$
\begin{aligned}
& \Delta H_{x}=E \delta_{x} \varepsilon_{x_{v}} \\
& \Delta H_{y}=E \delta_{y} \varepsilon_{y},
\end{aligned}
$$

где $\delta_{x}$ и $\delta_{y}-$ приведенные толщины оболочки. 
Подставляя значения $\varepsilon_{x}$ и $\varepsilon_{y}$ из (10) и (11) в зависимости (12) и (13), находим

$$
\begin{aligned}
& \Delta H_{x}=E \delta_{x}\left[\frac{\partial u}{\partial x}+\frac{\partial^{2} z}{\partial x^{2}} w+\frac{1}{2}\left(\frac{\partial w}{\partial x}\right)^{2}\right] \\
& \Delta H_{y}=E \delta_{y}\left[\frac{\partial v}{\partial y}+\frac{\partial^{2} z}{\partial y^{2}} w+\frac{1}{2}\left(\frac{\partial w}{\partial y}\right)^{2}\right] .
\end{aligned}
$$

Для исключения из уравнений (14) и (15) тангенциальных перемещений $u$ и $v$ рассмотрим граничные условия по этим перемещениям. Когда контур недеформируем, то в точках примыкания оболочки к контуру

$$
u=0, \quad v=0 .
$$

Приравнивая далее значения $\Delta H_{x}$ и $\Delta H_{y}$ по уравнениям (7), (8), (14) и (15), находим после интегрирования

$$
\begin{aligned}
& y_{0}(y) x+y_{1}(y)-\int d x \int q_{x} d x=E \delta_{x} \int \frac{\partial^{2} z}{\partial x^{2}} w d x+\frac{E \delta_{x}}{2} \int\left(\frac{\partial w}{\partial x}\right)^{2} d x \\
& X_{0}(x) y+X_{1}(x)-\int d y \int q_{y} d y=E \delta_{y} \int \frac{\partial^{2} z}{\partial y^{2}} w d y+\frac{E \delta_{y}}{2} \int\left(\frac{\partial w}{\partial y}\right)^{2} d y .
\end{aligned}
$$

В случае деформируемого контура в уравнения (16) и (17) войдут дополнительные члены, учитывающие его смещения.

Подставляя в уравнения (16) и (17) граничные значения координат и функции прогиба, находим значения неизвестных функций $y_{0}(y), y_{1}(y)$, $X_{0}(x)$ и $X_{1}(x)$.

Далее функция прогиба определяется из уравнения

$$
\begin{gathered}
{\left[y_{0}(y)-\int q_{x} d x\right] \frac{\partial^{2}(z+w)}{\partial x^{2}}+\left[X_{0}(x)-\int q_{y} d y\right] \frac{\partial^{2}(z+w)}{\partial y^{2}}+H_{x}^{0} \frac{\partial^{2} w}{\partial x^{2}}+} \\
+H_{y}^{0} \frac{\partial^{2} w}{\partial y^{2}}=q_{z}+q_{x} \frac{\partial(z+w)}{\partial x}+q_{y} \frac{\partial(z+w)}{\partial y} .
\end{gathered}
$$

Основная трудность при этом заключается в том, что в уравнения (16) и (17) для определения неизвестных функций $X_{0}(x)$ и $y_{0}(y)$ входит неизвестная пока функция прогиба w.

Гиперболический параболоид в случае недеформируемого эллиптического контура. Пусть поверхность оболочки (рис. 3) задана уравнением

$$
z=f_{x}\left(\frac{x}{a}\right)^{2}-f_{y}\left(\frac{y}{b}\right)^{2}
$$

Контур оболочки образован недеформируемой пространственной кривой, имеющей в плане форму эллипса

$$
1-\left(\frac{x}{a}\right)^{2}-\left(\frac{y}{b}\right)^{2}=0
$$

На оболочку действует равномерно распределенная нагрузка интенсивностью $q$. 


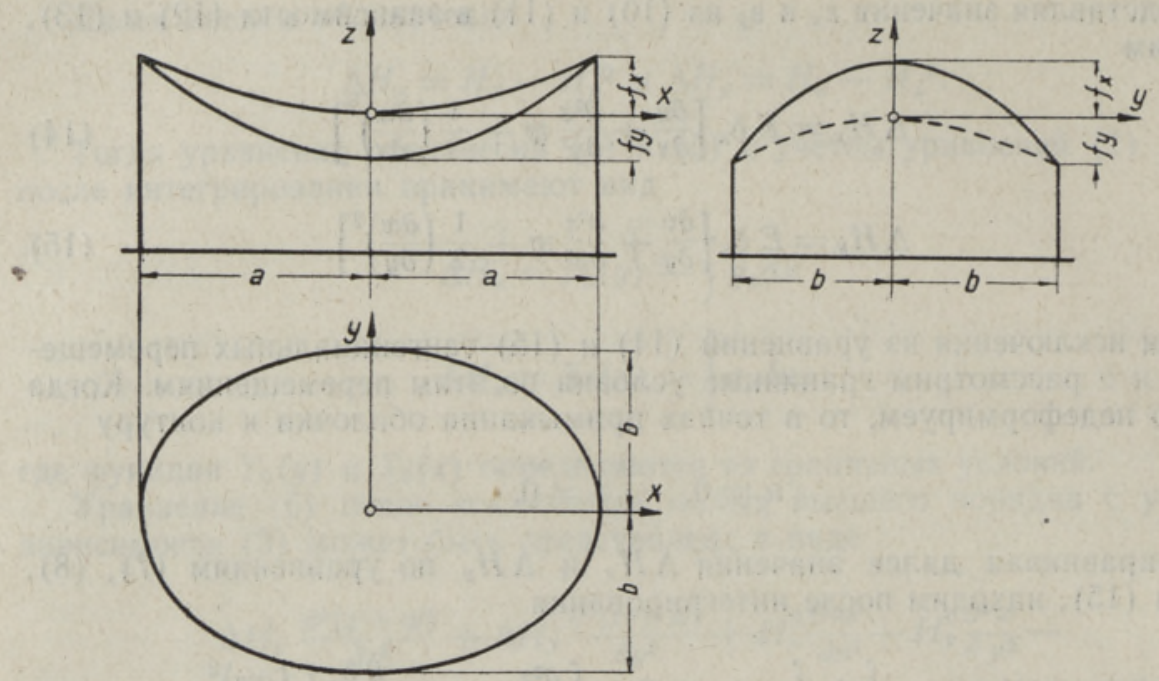

Рис. 3.

Функцией прогиба задаемся в виде

$$
w=\sum_{j=1,2, \ldots} w_{j}\left[1-\left(\frac{x}{a}\right)^{2}-\left(\frac{y}{b}\right)^{2}\right]^{j},
$$

удовлетворяющем граничным условиям и условию симметрии деформаций.

Согласно уравнению равновесия (3) в исходном состоянии имеем

$$
H_{y}^{0}=\frac{f_{x} b^{2}}{f_{y} a^{2}} H_{x}^{0}
$$

Поскольку усилия предварительного напряжения в разных направлениях прямо пропорциональны друг другу, то согласно уравнениям (1) и (2) они должны быть постоянными по всей поверхности оболочки.

Для вычисления интегралов уравнений (16) и (17) представим соответствующие функции в легко интегрируемом виде.

Функция прогиба (21) может быть представлена в виде двойных рядов

$$
w=\sum_{m=0,1}^{j} \sum_{j}(-1)^{m_{w}} C_{j}^{m}\left(\frac{x}{a}\right)^{2 m}\left[1-\left(\frac{y}{b}\right)^{2}\right]^{j-m}
$$

или

$$
w=\sum_{m=0,1}^{j} \sum_{j}(-1)^{m} w_{j} C_{j}^{m}\left(\frac{y}{b}\right)^{2 m}\left[1-\left(\frac{x}{a}\right)^{2}\right]^{j-m},
$$

где $C_{j,}^{m}-$ биномиальные коэффициенты Ньютона.

Для первых производных находим

$$
\begin{aligned}
& \frac{\partial w}{\partial x}=-\sum_{j=1,2} \frac{2 j x}{a^{2}} w_{j}\left[1-\left(\frac{x}{a}\right)^{2}-\left(\frac{y}{b}\right)^{2}\right]^{j-1} \\
& \frac{\partial w}{\partial y}=-\sum_{j=1,2} \frac{2 j y}{b^{2}} w_{j}\left[1-\left(\frac{x}{a}\right)^{2}-\left(\frac{y}{b}\right)^{2}\right]^{j-1} .
\end{aligned}
$$


После возведения в квадрат имеем

$$
\begin{aligned}
& \left(\frac{\partial w}{\partial x}\right)^{2}=\sum_{m=0,1}^{j+k-2} \sum_{j} \sum_{k}(-1)^{m} \frac{4 j k w_{j} w_{k}}{a^{2}} C_{j+k-2}^{m}\left(\frac{x}{a}\right)^{2(m+1)}\left[1-\left(\frac{y}{b}\right)^{2}\right]^{j+k-(m+2)} \\
& \left(\frac{\partial w}{\partial y}\right)^{2}=\sum_{m=0,1}^{j+k-2} \sum_{j} \sum_{k}(-1)^{\frac{m}{\frac{4 j k w_{j} w_{k}}{b^{2}}} C_{j+k-2}^{m}\left(\frac{y}{b}\right)^{2(m+1)}\left[1-\left(\frac{x}{a}\right)^{2}\right]^{j+k-(m+2)} .}
\end{aligned}
$$

Интегрирование дает

$$
\begin{gathered}
\int \frac{\partial^{2} z}{\partial x^{2}} w d x+\frac{1}{2} \int\left(\frac{\partial w}{\partial x}\right)^{2} d x=\frac{2 f_{x}}{a} \sum_{m=0,1}^{j} \sum_{j}(-1)^{m} C_{j}^{m} \frac{w_{j}}{2 m+1}\left(\frac{x}{a}\right)^{2 m+1} \times \\
\times\left[1-\left(\frac{y}{b}\right)^{2}\right]^{j-m}+2 \sum_{m=0,1}^{j+k-2} \sum_{j} \sum_{k}(-1)^{m} \frac{j k w_{j} w_{k}}{(2 m+3) a} \times \\
\times C_{j+k-2}^{m}\left(\frac{x}{a}\right)^{2 m+3}\left[1-\left(\frac{y}{b}\right)^{2}\right]^{j+k-(m+2)}
\end{gathered}
$$

$\int \frac{\partial^{2} z}{\partial y^{2}} w d y+\frac{1}{2}\left(\frac{\partial w}{\partial y}\right)^{2} d y=-\frac{2 f_{y}}{b} \sum_{m=0,1}^{j} \sum_{j}(-1)^{m} C_{j}^{m} \frac{w_{j}}{2 m+1}\left(\frac{y}{b}\right)^{2 m+1} \times$

$$
\begin{gathered}
\times\left[1-\left(\frac{x}{a}\right)^{2}\right]^{j-m}+2 \sum_{m=0,1}^{j+k-2} \sum_{j} \sum_{k}(-1)^{m} \frac{j k w_{j} w_{k}}{(2 m+3) b} C_{j+k-2}^{m} \times \\
\times\left(\frac{y}{b}\right)^{2 m+3}\left[1-\left(\frac{x}{a}\right)^{2}\right]^{j+k-(m+2)}
\end{gathered}
$$

Подставляя найденные интегралы в уравнения (16) и (17), находим: при $x=0: \quad y_{1}(y)=0$,

нри $\left(\frac{x}{a}\right)^{2}=1-\left(\frac{y}{b}\right)^{2}$ :

$$
\begin{aligned}
& y_{0}(y)=\frac{2 E \delta_{x}}{a^{2}}\left\{f_{x} \sum_{m=0,1}^{j} \sum_{j} \frac{(-1)^{m}}{2 m+1} C_{j}^{m} w_{j}\left[1-\left(\frac{y}{b}\right)^{2}\right]^{j}+\right. \\
& \left.+\sum_{m=0,1}^{j+k} \sum_{j} \sum_{k} \frac{(-1)^{m}}{2 m+3} C_{j+k-2}^{m} w_{j} w_{k}\left[1-\left(\frac{y}{b}\right)^{2}\right]^{j+k-1}\right\}
\end{aligned}
$$

при $y=0: \quad X_{1}(x)=0$,

гри $\left(\frac{y}{b}\right)^{2}=1-\left(\frac{x}{b}\right)^{2}$ :

$$
\begin{gathered}
X_{0}(x)=\frac{2 E \delta_{y}}{b^{2}}\left\{-f_{y} \sum_{m=0,1}^{j} \sum_{j} \frac{(-1)^{m}}{2 m+1} C_{j}^{m} w_{j}\left[1-\left(\frac{x}{a}\right)^{2}\right]^{j}+\right. \\
+\sum_{m=0,1}^{j+k-2} \sum_{j} \sum_{k} \frac{(-1)^{m}}{2 m+3} C_{j+k-2}^{m} w_{i} w_{k}\left[1-\left(\frac{x}{a}\right)^{2}\right]^{j+k-1}
\end{gathered}
$$


Вторые производные от функции прогиба представим в внде:

$\frac{\partial^{2} w}{\partial x^{2}} \sum_{n=0,1}^{i-2} \sum_{i}(-1)^{n} C_{i-2}^{n} \frac{w_{i}}{a^{2}}\left(\frac{x}{a}\right)^{n}\left[1-\left(\frac{y}{b}\right)^{2}\right]^{i-2-n}\left[1-(2 i-1)\left(\frac{x}{a}\right)^{2}-\left(\frac{y}{b}\right)^{2}\right]$

$\frac{\partial^{2} w}{\partial y^{2}}=\sum_{n=0,1}^{i-2} \sum_{i}(-1)^{n} C_{i-2}^{n} \frac{w_{i}}{b^{2}}\left(\frac{y}{b}\right)^{n}\left[1-\left(\frac{x}{a}\right)^{2}\right]^{i-2-n}\left[1-\left(\frac{x}{a}\right)^{2}-(2 i-1)\left(\frac{y}{b}\right)^{2}\right]$.

Далее подставим найденные коэффициенты интегрирования и производные в уравнение (18). В результате имеем уравнение равновесия в виде

$$
\begin{aligned}
& \frac{4 E \delta_{x}}{a^{4}}\left\{f_{x}-\sum_{n=0,1}^{i-2} \sum_{i}(-1)^{n} C_{i-2}^{n} w_{i}\left(\frac{x}{a}\right)^{2 n}\left[1-\left(\frac{y}{b}\right)^{2}\right]^{i-2-n} \times\right. \\
& \times\left.\left.-(2 i-1)\left(\frac{x}{a}\right)^{2}-\left(\frac{y}{b}\right)^{2}\right]\right\}\left\{f_{x} \sum_{m=0,1}^{j} \sum_{j} \frac{(-1)^{m}}{2 m+1} C_{j}^{m} w_{j}\left[1-\left(\frac{y}{b}\right)^{2}\right]^{j}+\right. \\
&\left.+\sum_{m=0,1}^{j+k-2} \sum_{j} \sum_{k} \frac{(-1)^{m}}{2 m+3} C_{j+k-2}^{m} j k w_{j} w_{k}\left[1-\left(\frac{y}{b}\right)^{2}\right]^{j+k-1}\right\}- \\
&-\frac{4 E \delta_{y}}{b^{4}}\left\{f_{y}+\sum_{n=0,1}^{i-2} \sum_{i}(-1)^{n} C_{i-2}^{n} w_{i}\left(\frac{y}{b}\right)^{2 n}\left[1-\left(\frac{x}{a}\right)^{2}\right]^{i-2-n} \times\right. \\
&\left.\times\left[1-\left(\frac{x}{a}\right)^{2}-(2 i-1)\left(\frac{y}{b}\right)^{2}\right]\right\}\left\{f_{y} \sum_{m=0,1}^{j} \sum_{j} \frac{(-1)^{m}}{2 m+1} C_{j}^{m} w_{j}\left[1-\left(\frac{x}{a}\right)^{2}\right]^{j}+\right. \\
&\left.+\sum_{n=0,1}^{j+k-2} \sum_{j} \sum_{k} \frac{(-1)^{m}}{2 m+3} C_{j+k-2}^{m} j k w_{j} w_{k}\left[1-\left(\frac{x}{a}\right)^{2}\right]^{j+k-1}\right\}+ \\
&+\frac{2 H_{x}^{0}}{a^{2}} \sum_{n=0,1}^{i-2} \sum_{i}(-1)^{n} C_{i-2}^{n} w_{i}\left\{( \frac { x } { a } ) ^ { 2 n } [ 1 - ( \frac { y } { b } ) ^ { 2 } ] ^ { i - 2 - n } \left[1-(2 i-1)\left(\frac{x}{a}\right)^{2}-\right.\right. \\
&\left.\left.-\left(\frac{y}{b}\right)^{2}\right]+\frac{f_{x}}{f_{y}}\left(\frac{y}{b}\right)^{2 n}\left[1-\left(\frac{x}{a}\right)^{2}\right]^{i-2-n}\left[1-\left(\frac{x}{a}\right)^{2}-(2 i-1)\left(\frac{y}{b}\right)^{2}\right]\right\}-q=0 .
\end{aligned}
$$

Уравнение (35) может быть решено, например, методом Бубнова-Галеркина. Когда функция прогиба аппроксимируется $s$ членами, то после интегрирования по условиям ортогональности получим систему с $s$ кубическими уравнениями относительно коэффициентов $w_{i}$.

Некоторые соображения по учету нелинейности задачи. В приведенных выше формулах приняты во внимание как геометрическая нелинейность, так и нелинейность, обусловленная предварительным напряжением системы. Последняя заключается в учете конечных смещений в уравнениях равновесия при выводе уравнения (18) и характеризуется членами, содержащими вторые производные от смещений ш. Геометрическая์ же нелинейность учтена в значениях функций $y_{0}(y)$ и $X_{0}(x)$ и характеризуется членами, содержащими квадраты первых производных от смещений ш в формулах (16) и (17). 
Легко убедиться, что неучет смещений в уравнениях равновесия и неучет геометрической нелинейности приводят к погрешности одинакового порядка и характера. С другой стороны, значение этой погрешности увеличивается с увеличением соотношений $\frac{w_{\max }}{f_{x}}$ и $\frac{w_{\max }}{f_{y}}$, т. е. при увеличении деформативности и пологости оболочки.

Для оценки погрешности решения в линейной постановке в каждом конкретном случае можно решить линейный вариант уравнения (18), а затем сравнить полученный максимальный прогиб со стрелой кривизны оболочки. При этом функция прогиба может быть аппроксимирована небольшим количеством членов.

\section{ЛИТЕРАТУ РА}

1. Муштари Х. М., Галия и в К. 3., Нелинейная теория упругих оболочек, Қазань, 1957.

2. Висячие покрытия (Тр. совещ. по исслед. и внедр. висячих покрытий), М., 1962.

3. К а ч ур и н В. К.; Теория висячих систем, Л.-М., 1962.

4. Л аул Х. Х.. Кульбах В. Р., Сумбак А. А., Тр. Таллинск. политехн: ин-та, Cep. A, № 184, 3-22 (1961).

5. Кульб ах В. Р., К вопросу расчета висячих покрытий отрицательной кривизны, имеющих в плане форму эллипса, Тр. Таллинск. политехн. ин-та, Сер. А. (В печати).

6. E r a s, E $1 \mathrm{z}$ e, Bauplanung und Bautechnik, H. 7., 349-354 (1961).

таллинский политехнический институт

\section{Поступила в редакцию} 16/III 1965

\section{KULBACH}

\section{AINULT TOMBELE TÖOTAVATE NEGATIIVSE KÖVERUSEGA LAMEDATE KOORIKUTE ARVUTAMISEST}

Artiklis vaadeldakse ainult tõmbele töötavaid negatiivse kõverusega lamedaid koorikuid. Saadud tulemused on rakendatavad ortogonaalsete trosside vorrguga rippkatuste arvutamisel.

Vōrrandite tuletamisel on arvestatud nii geomeetrilist mittelineaarsust kui ka sellist mittelineaarsust, mis on tingitud lōplike paigutuste arvestamisest ettepingutatud süsteemis. Saadud vôrrandite lahendamiseks soovitatakse kasutada Bubnovi-Galjorkini meetodit.

\section{KULBACH}

\section{ON THE CALCULATION OF PLANE CASINGS OF A NEGATIVE CURVATURE, RESISTENT TO TENSION}

The present article deals with the calculation of plane casings of a negative curvature which are resistent to forces of tension only. The received solution can be used for a planning of preliminarily stressed hanging roofs with a network of orthogonal lines.

At the deduction of equations, on the one hand, the geometrical non-linear factor is taken into account and on the other - the non-linear factor, depending on the final locations in the equilibrated equations. The analysis shows that both the non-linear factors influence the final result in the same way and that the neglect of these factors leads to errors of identical nature and order.

The solution of this problem results in a differential equation of the second order concerning the function of the flexure $w$; the coefficients of the equation depend on the function of the flexure and it is possible to determine these coefficients by means of extreme conditions. For the purpose of the solution of the equation, the author of the present article advises to approximate the function of the flexure in form of a special line of powers, and to use in future the method of Bubnoff-Galyorkin. As an example, the article examines a case of a hyperbolic paraboloid with an elliptical outline. 